\title{
Efeitos Ambientais e Genéticos sobre o Ganho em Peso Diário de Bovinos Nelore no Estado da Paraíba'
}

\author{
José Lindenberg Rocha Sarmento ${ }^{2}$, Edgard Cavalcanti Pimenta Filho ${ }^{3}$, Maria Norma Ribeiro ${ }^{4}$, \\ Raimundo Martins Filho 5
}

\begin{abstract}
RESUMO - Registros de 6.674 pesos de bovinos da raça Nelore nascidos entre 1975 e 1990 em duas fazendas no Estado da Paraíba foram utilizados para estudar efeitos ambientais e estimar parâmetros genéticos das características ganhos em peso diário do nascimento aos 205, dos 205 aos 365 e dos 365 aos 550 dias de idade. Os efeitos ambientais foram estudados usando-se o programa SAS ${ }^{\circledR}$. O modelo incluiu o efeito aleatório de touro e os efeitos fixos de ano-mês-sexo, fazenda e idade da vaca como covariável linear e quadrática. Os componentes de (co)variância usados para estimar os coeficientes de herdabilidade direta e materna foram obtidos pelo Método da Máxima Verossimilhança Restrita, usando o programa MTDFREML sob modelo animal. Todos os efeitos incluídos no modelo foram significativos, exceto o efeito de fazenda para o ganho diário do nascimento à desmama. As médias estimadas foram de $0,575 \pm 0,089$, $0,231 \pm 0,114$ e 0,283 $\pm 0,110 \mathrm{~kg}$ para ganhos em peso diário do nascimento aos 205, dos 205 aos 365 e dos 365 aos 550 dias de idade, respectivamente. As herdabilidades estimadas para efeito direto foram de 0,12, 042 e 0,21 e, para efeito materno, de 0,29 , 0,00 e 0,00 , na mesma ordem. Os coeficientes de herdabilidade indicam que a seleção baseada no ganho em peso pós-desmama até um ano de idade pode proporcionar considerável ganho genético.
\end{abstract}

Palavras-chave: bovinos de corte, crescimento, Nordeste do Brasil, parâmetros genéticos

\section{Genetic and Environmental Effects on Nellore Cattle Growth in Paraiba}

\begin{abstract}
Records on 6,674 Nellore cattle weight, born from 1975 to 1990 in two farms located in Paraiba State were used to study environmental effects and to estimate genetic parameters for average daily gains from birth to 205 days, from 205 to 365 days and from 365 to 550 days. The $S A S^{\circledR}$ program was used to study the environmental effects. The model included the random effect of sire and the fixed effects of year-month-sex, farm and age of the cow at calving as linear and quadratic covariate. The (co)variance components used to estimate direct and maternal heritabilities were obtained by Derivative Free Restricted Maximum Likelihood using the MTDFREML program, under an animal model. All effects used were significant, but the farm effect for pre-weaning daily gain. The estimated means for average daily gains were $0.575 \pm 0.089,0.231 \pm 0.114$ and $0.283 \pm 0.110 \mathrm{~kg}$ for pre-weaning daily gain, daily gain from 205 to 365 days and daily gain from 365 to 550 days of age, respectively. The heritabilities estimated for direct effect were 0.12 , 0.42 and 0.21 and for maternal effect were $0.29,0.00$ and 0.00 . The estimates of heritability show that selection based on daily gain from 205 days to yearling could allow considerable genetic gain.
\end{abstract}

Key Words: beef cattle, genetic parameters, growth, Northeast of Brazil

\section{Introdução}

O Brasil, por apresentar grande extensão territorial e considerável efetivo de população bovina (aproximadamente 150.276.778 cabeças - Anualpec, 1999), dispõe de condições suficientes para suprir as necessidades internas de demanda, como também contribuir de maneira significativa para a exportação e o abastecimento do mercado externo de carne bovina.

$\mathrm{O}$ aumento de produtividade pode ser obtido com o auxílio da identificação e multiplicação dos melhores genótipos, através da correta segregação dos fatores genéticos e ambientais que interferem no crescimento dos animais, visando obter programas adequados de melhoramento genético.

O crescimento rápido é uma característica desejável em bovinocultura de corte, pois animais que têm maior capacidade de crescimento precisam de menor período de tempo para atingir a idade de abate. Dessa forma, os ganhos médios diários devem ser estudados e incluídos em programas de seleção.

Estudando os pesos e ganhos em pesos de bovinos

\footnotetext{
${ }_{1}^{1}$ Parte do trabalho de graduação apresentado à UFPB, Areia - PB, pelo primeiro autor. Apoio financeiro: CNPq.

${ }_{2}^{2}$ Mestrando em Zootecnia do DZO/UFV e bolsista do CNPq. Viçosa-MG, CEP: 36571-000. E.mail: lindeb@bol.com.br

3 Professor Adjunto do Departamento de Zootecnia da UFPB, Areia-PB, CEP: 58397-000. E.mail: edgard@cca.ufpb.br

4 Professora Adjunto do Departamento de Zootecnia da UFRPE. E.mail: mn.ribeiro@uol.com.br

${ }^{5}$ Professor Adjunto do Departamento de Zootecnia da UFC. E.mail: martins@ufc.br
} 
zebus no Estado do Ceará, Martins Filho et al (1997) verificaram que a idade da vaca ao parto influenciou significativamente apenas o ganho de peso diário do nascimento à desmama. Ainda no mesmo estudo, os autores constataram ganho médio de $450 \mathrm{~g}$ por dia em bovinos Nelore na fase de pré-desmame; na fase de pós-desmama, observaram ganho médio diário até os 365 dias de $310 \mathrm{~g}$ e, dos 365 aos 550 dias, de $260 \mathrm{~g}$. Muniz \& Queiroz (1998) avaliaram o ganho médio de peso de bezerros Nelore e seus mestiços e verificaram ganho médio de $630 \mathrm{~g}$ por dia do nascimento à desmama. Lôbo et al (1995) encontraram ganho médio diário de $288 \mathrm{~g}$ do desmame aos 365 dias e de $389 \mathrm{~g}$ dos 365 aos 550 dias de idade.

Verificando a influência de efeitos genéticos e de meio sobre o crescimento de bovinos Nelore, Martins et al. (1998) constataram que rebanho, mês e ano de nascimento da cria, sexo e regime alimentar se constituíram fonte de variação significativa para o ganho diário do nascimento à desmama e que a idade da vaca ao parto não influenciou significativamente essa característica.

Biffani (1997), estudando características de crescimento em bovinos Nelore na região Nordeste, encontrou efeito significativo de sexo, ano de nascimento e fazenda para o ganho diário da desmama aos 365 dias e dos 365 aos 550 dias de idade. O efeito de estação de nascimento foi significativo apenas para o ganho diário da desmama aos 365 dias de idade, e a idade da vaca ao parto não influenciou as características estudadas.

A herdabilidade de determinada característica, considerada a base fundamental dos programas de melhoramento genético animal, é definida como a proporção da variância fenotípica devida à variância genética aditiva. Vários autores têm estimado a herdabilidade para os ganhos diários, entre eles Martins et al. (1998), que encontraram herdabilidade para ganho de peso diário do nascimento à desmama de 0,35; e Martins Filho et al. (1997), que verificaram herdabilidade de $0,17,0,13$ e 0,13 para ganho diário do nascimento à desmama, da desmama aos 365 e dos 365 aos 550 dias de idade, respectivamente.

$\mathrm{O}$ ambiente materno pode influenciar o crescimento do bezerro tanto na fase pré-natal como na pós-natal. Nesta última, a influência materna estará em maior dependência do manejo empregado. Segundo Sousa et al. (1999), para aumentar o ganho genético em programas de seleção de características influenciadas pelo efeito materno, é necessário obter mais informações sobre esse efeito. Se os efeitos genéticos maternos são também importantes, mas não são considerados nos modelos, as estimativas de herdabilidade podem estar viciadas e a eficiência da seleção pode ser reduzida (Snyman et al., 1995).

Este trabalho foi conduzido com os objetivos de estudar os efeitos ambientais e determinar os parâmetros genéticos direto e materno que influenciaram o ganho médio diário de peso de bovinos Nelore criados no Estado da Paraíba.

\section{Material e Métodos}

Os dados foram provenientes de animais com registro no Controle de Desenvolvimento Ponderal realizado pela Associação Brasileira dos Criadores de Zebu no Estado da Paraíba, referentes a bovinos de corte da raça Nelore.

As informações coletadas durante 15 anos (1975 1990) provieram de duas fazendas no Estado da Paraíba, uma localizada no Município de São Miguel de Taipu, Fazenda Oiteiro, e a outra no Município de Gurinhém, Fazenda Morcego, e são o resultado de pesagens trimestrais efetuadas até os 18 meses de idade; apenas o peso ao nascer foi efetuado pelo criador. De maneira generalizada, a região apresenta clima típico de caatinga litorânea nordestina.

De modo geral, os animais permaneceram no campo durante todo o ano em regime exclusivo de pastejo com suplementação mineral, à vontade. Os animais tinham livre acesso a pastagem nativa, nativa melhorada e pastagem cultivada, a qual representava a única forma de alimentação. Sabe-se, no entanto, que em períodos de escassez de alimentos os criadores faziam suplementação dos animais com volumosos de várias origens, de acordo com a época e a propriedade envolvida.

Os dados gerados pelo Controle de Desenvolvimento Ponderal da ABCZ foram usados para estimar os parâmetros fenotípicos e genéticos relativos aos ganhos médios diários do nascimento à desmama (GPND), da desmama aos 365 dias de idade (GPDA) e dos 365 dias aos 550 dias de idade (GPAS); os pesos usados para calcular os ganhos médios foram todos ajustados a essas idades-padrão, de acordo com fórmulas apresentadas por Lôbo (1992). Os ganhos médios diários, citados anteriormente, foram calculados pela diferença entre o peso posterior e o anterior, divido pelo número de dias contido no período compreendido entre as duas pesagens. 
Os dados foram editados de modo que se consideraram apenas animais nascidos entre 1975 e 1990; somente animais com efeitos fixos presentes (fazenda, ano e mês de nascimento, sexo e raça); somente animais com efeitos aleatórios presentes (pai e mãe); e animais nascidos de mães com idade dentro da amplitude considerada normal. Foi também definido que os touros com mais de seis filhos no rebanho e grupos de efeito ano-mês-sexo com pelo menos três animais foram mantidos no arquivo geral. Alguns possíveis animais com pesos fora da amplitude considerada normal foram eliminados. Após a edição final, o arquivo para análise constava de 6.647 registros, totalizando 8.943 animais na matriz de numeradores dos coeficientes de parentesco.

As análises foram divididas em duas etapas: na primeira, buscou-se estudar os efeitos ambientais para cada característica, e, na segunda, estimar os parâmetros genéticos.

As análises dos efeitos ambientais foram feitas pelo procedimento GLM do programa SAS® (1996), considerando-se a opção RANDOM, que trata o efeito de reprodutor como aleatório, utilizando o modelo estatístico a seguir:

$Y_{i j k l}=\mu+r_{i}+F_{j}+A M S_{k}+b_{1}\left(I_{i j k l}-\bar{I}\right)+b_{2}\left(I_{i j k l}-\bar{I}\right)^{2}+\varepsilon_{i j k l}$

em que: $Y_{i j k l}=$ GPND, GPDA e GPAS do enésimo filho do reprodutor $i$, da fazenda $j$ e pertencente ao efeito ano-mês-sexo $\mathrm{k} ; \mu=$ média geral para as características estudadas; $\mathrm{r}=$ efeito aleatório do reprodutor $\mathrm{i} ; \mathrm{F}_{\mathrm{j}}=$ efeito fixo da fazenda $\mathrm{j} ; \mathrm{AMS}_{\mathrm{k}}=$ efeito de ano-mês-sexo $\mathrm{k}$, formado pelos animais nascidos no mesmo ano, no mesmo mês e do mesmo sexo; $\mathrm{I}_{\mathrm{ijkl}}=$ efeito da idade da vaca ao parto, como covariável; $b_{1}=$ coeficiente de regressão linear para a idade da vaca; $b_{2}=$ coeficiente de regressão quadrático para a idade da vaca; $\mathrm{e}_{\mathrm{ijkl}}=$ erro aleatório independentemente distribuído com média zero e variância $I \sigma^{2}$.

Foram considerados como fixos os efeitos anomês-sexo e fazenda, bem como o efeito aleatório de reprodutor para cada característica analisada. A idade da vaca ao nascimento da cria foi utilizada como co-variável (efeitos linear e quadrático).

As estimativas dos componentes de (co)variância e dos parâmetros genéticos foram obtidas utilizandose o aplicativo MTDFREML, descrito por Boldman et al. (1995), que utiliza a metodologia da Máxima Verossimilhança Restrita livre de derivadas, sob um modelo animal. Foi utilizado como critério de convergência a variância dos valores do simplex $\left(-2 \log _{\mathrm{e}} \mathrm{de}\right.$ verossimilhança) inferior a $10^{-9}$. Após a convergência, o programa foi reiniciado, usando as estimativas obtidas anteriormente como valores iniciais. Esse procedimento foi repetido até que a diferença entre as estimativas das duas últimas convergências fosse menor que $10^{-5}$.

Usando uma notação matricial, as análises foram conduzidas utilizando-se o seguinte modelo:

$$
Y=\mathrm{X} \beta+\mathrm{Z}_{1} d+\mathrm{Z}_{2} m+\varepsilon
$$

em que: $Y=$ vetor das observações n $\mathrm{x} 1 ; X=$ matriz de incidência dos efeitos fixos $n \times f ; \beta=$ vetor dos efeitos fixos $\mathrm{f} \times 1 ; \mathrm{Z}_{1}=$ matriz de incidência dos efeitos genéticos aditivos diretos $\mathrm{n} \times \mathrm{N} ; d=$ vetor dos efeitos genéticos aditivos diretos $\mathrm{N} \times 1 ; \mathrm{Z}_{1}=$ matriz de incidência dos efeitos genéticos aditivos maternos $\mathrm{n} \times \mathrm{N} ; m=$ vetor dos efeitos genéticos aditivos maternos $\mathrm{N} \times 1 ; \varepsilon=$ vetor dos efeitos residuais $\mathrm{n} \times 1$. Neste caso, $\mathrm{n}=$ número de observações; $\mathrm{f}=$ número de níveis de efeitos fixos; e $\mathrm{N}=$ número de animais com observação.

As pressuposições assumidas deste modelo, em relação aos vetores $y, d, m$ e $e$, são de que possuem distribuição normal com $\mathrm{E}(y)=\mathrm{X} \beta, \mathrm{E}(d)=\mathrm{E}(m)=$ $\mathrm{E}(e)=0, \operatorname{Var}(y)=Z_{1} A Z_{1}^{\prime} \sigma_{a}^{2}+Z_{2} Z_{2}^{\prime} \sigma_{m}^{2}+R$, $\operatorname{Var}(d)=A \sigma_{d}^{2}, \operatorname{Var}(m)=A \sigma_{m}^{2}$ e $\operatorname{Var}(\varepsilon)=I \sigma_{\varepsilon}^{2}$, sendo: $\mathrm{A}=$ matriz de parentesco, $\mathrm{I}=$ matriz identidade, $\mathrm{R}$ = matriz de variâncias e covariâncias residuais, $\sigma_{a}^{2}=$ componente de variância genético aditivo direto, $\sigma_{m}^{2}=$ componente de variância genético aditivo materno e $\sigma_{\varepsilon}^{2}=$ componente de variância residual.

Foram considerados os mesmos efeitos fixos usados na análise anterior.

\section{Resultados e Discussão}

Os ganhos médios diários de pesos obtidos foram de $0,575 \pm 0,089 \mathrm{~kg}, 0,231 \pm 0,114 \mathrm{~kg} \mathrm{e} 0,283 \pm 0,110$ $\mathrm{kg}$, com coeficiente de variação igual a 15,55\%, $49,63 \%$ e $39,10 \%$, respectivamente, para GPND, GPDA e GPAS. As médias obtidas para os ganhos em peso diários estão próximas das verificadas por Lôbo et al. (1995), Martins Filho et al. (1997) e Biffani (1997), todos estudando animais da raça Nelore criados na região Nordeste.

Todos os efeitos estudados foram significativos $(\mathrm{P}<0,05)$, exceto o efeito de fazenda para o GPND (Tabela 1). As diferentes formas de manejo, próprias 
de cada fazenda, além das diferenças climáticas e de origem genética do rebanho, não influenciaram significativamente o GPND, o que não aconteceu com os demais ganhos em peso diário, em que todas as variáveis estudadas apresentaram efeito significativo. A influência materna no período do nascimento à desmama foi alta porque, nesse período, o bezerro depende quase exclusivamente dos cuidados da mãe. Esse fato pode ter contribuído para diminuir as prováveis diferenças de meio existentes em cada fazenda, tornando esse efeito não significativo.

A Fazenda Morcego apresentou, para os três ganhos de peso diário, média superior à verificada na Fazenda Oiteiro, provavelmente devido a melhores condições de manejo, facilitadas pelo menor número de animais, ou, mesmo, em razão de os animais dela serem geneticamente superiores.

O efeito ano-mês-sexo apresentou-se significativo sobre os três ganhos em pesos diários. As diferenças proporcionadas pelas condições edafoclimáticas específicas de cada região, como, por exemplo, a estacionalidade na produção de alimentos em termos qualitativos e quantitativos, pode ter contribuído, direta ou indiretamente, para as diferenças existentes.

A idade da vaca ao parto tomada como covariável linear e quadrática apresentou efeito significativo para os ganhos do nascimento à desmama e da desmama a um ano, contrariando os resultados obti- dos por Martins Filho et al. (1997) e Biffani (1997). Para o ganho de peso de ano a sobreano esta variável não se mostrou significativa, corroborando os achados de Biffani (1997).

A influência da idade da mãe está relacionada com a habilidade materna desde a fase pré-natal, em razão das diferenças placentárias, até a fase pós-natal, devido aos cuidados proporcionados e à produção de leite da mãe. Essa influência é evidenciada até a desmama, pelo fato de o bezerro depender quase exclusivamente da mãe. A influência materna, nesta fase, foi responsável por $46,9 \%$ da variação fenotípica total. A partir da desmama, o bezerro torna-se mais independente e a influência desse efeito tende a diminuir, chegando a desaparecer totalmente de um ano a um ano e meio, como foi verificado neste trabalho. A contribuição materna para o período da desmama a um ano de idade foi de $11,23 \%$ da variação total e de apenas, $0,6 \%$ para o período de um ano a um ano e meio de idade, não sendo mais significativo. $\mathrm{O}$ resultado verificado neste estudo pode ser justificado pelo fato de o bezerro ter permanecido ao pé da mãe até idades mais avançadas, não sendo desmamado entre seis e sete meses de idade, e, também, devido às condições de criação - o animal permaneceu no campo o ano todo. Assim, o desempenho do bezerro sofreu mais influência do efeito materno. A partir de um ano e um ano e meio de idade, o animal passou a expressar sua própria capacidade de crescimento.

Tabela 1 - Resumo da análise de variância para ganho de peso diário do nascimento ao desmame (GPND), da desmama aos 365 dias (GPDA) e dos 365 aos 550 dias de idade (GPAS)

Table 1 - Summary of variance analysis for average daily gains from birth to weaning (WBWG), from weaning to 365 days (365WWG) and from 365 to 550 days of age (550YWG)

\begin{tabular}{|c|c|c|c|c|c|c|}
\hline \multirow{3}{*}{$\begin{array}{l}\text { Fontes de variação } \\
\text { Source of variation }\end{array}$} & \multicolumn{2}{|c|}{$\begin{array}{l}\text { GL } \\
D F\end{array}$} & \multicolumn{4}{|c|}{$\begin{array}{c}\text { Quadrado médio } \\
\text { Mean square }\end{array}$} \\
\hline & GPND & GPDA & GPAS & GPND & GPDA & GPAS \\
\hline & $W B W G$ & $365 W W G$ & $550 Y W G$ & $W B W G$ & $365 W W G$ & $550 Y W C$ \\
\hline Touro & 64 & 59 & 37 & $0,031^{*}$ & $0,047^{*}$ & $0,019^{*}$ \\
\hline $\begin{array}{l}\text { Sire } \\
\text { Ano-mês-sexo } \\
\text { Year-month-sex }\end{array}$ & 164 & 141 & 69 & $0,073^{*}$ & $0,205^{*}$ & $0,129^{*}$ \\
\hline Fazenda & 1 & 1 & 1 & 0,001 & $0,054^{*}$ & $0,140^{*}$ \\
\hline $\begin{array}{l}\text { Farm } \\
\text { Idade da vaca ao parto (linear) } \\
\text { Age of cow at calving (linear) }\end{array}$ & 1 & 1 & 1 & $0,884^{*}$ & $0,076^{*}$ & 0,003 \\
\hline $\begin{array}{l}\text { Idade da vaca ao parto (quadrático) } \\
\text { Age of cow at calving (quadratic) }\end{array}$ & 1 & 1 & 1 & $0,884^{*}$ & $0,050^{*}$ & 0,002 \\
\hline $\begin{array}{l}\text { Erro } \\
\text { Error }\end{array}$ & 5235 & 3477 & 890 & 2463,91 & 0,0132 & 0,012 \\
\hline
\end{tabular}

R. Bras. Zootec., v.32, n.2, p.325-330, 2003 
Dessa forma, pode-se dizer que o animal foi totalmente desmamado depois de um ano de idade.

As estimativas de herdabilidade para GPND, GPDA e GPAS foram de baixa, alta e média magnitude, respectivamente (Tabela 2), indicando que o valor fenotípico pode ser utilizado como estimador satisfatório do valor genético aditivo direto; conseqüentemente, espera-se obter ganho genético através da seleção, desde que esta ocorra nas idades mais avançadas, ou seja, após a desmama. As herdabilidades estimadas para o ganho de peso nas várias idades foram superiores às estimadas por Martins Filho et al. (1997) e inferior à obtida por Martins et al. (1998) para o GPND.

A contribuição do efeito materno na variação fenotípica total foi de magnitude média para o GPND, justificando sua inclusão no modelo e permitindo maior acurácia na estimativa dos parâmetros genéticos. Para o GPND, o efeito materno teve maior contribuição na variação fenotípica total (29\%). Esse resultado indica que a seleção nesse período não trará resposta satisfatória. Em se tratando do GPDA e GPAS, o efeito materno teve pouca importância, sugerindo que a variação fenotípica para essas características está associada ao efeito genético aditivo direto.

As correlações estimadas entre os ganhos médios diários de pesos foram positivas e de baixa magnitude (Tabela 3), indicando que grande parte dos genes responsáveis pela expressão da característica GPND não continua atuando na expressão das demais. Assim, animais que ganham mais peso do nascimento à desmama poderiam não ser os mesmos a ganhar mais peso nas idades subseqüentes. Com isso, a resposta correlacionada poderá não proporcionar resultados desejáveis, embora a correlação seja favorável à seleção. A correlação genética entre GPDA e GPAS encontrada neste estudo foi maior que a obtida por Biffani (1997), que foi de 0,09 .

As correlações genéticas entre o efeito genético aditivo direto e o materno foram negativas, de baixa a alta magnitude (Tabela 2), indicando antagonismo entre os efeitos dos genes para potencial de crescimento da cria e habilidade materna da mãe, sendo mais marcante para o GPND (-0,77). Correlação menor e negativa $(-0,22)$ foi obtida por Eler et al. (1996). Magnabosco et al. (1996) enfatizaram que as altas e negativas correlações genéticas encontradas entre o efeito direto e o materno seriam devidas mais a inadequados modelos estatísticos usados na análise do que a uma causa biológica. As correlações estimadas neste estudo foram negativas e maiores do que as verificadas por Eler et al. (2000) sob modelo que, também, não incluiu a interação touro x rebanho. Segundo esses autores a não inclusão do efeito da interação touro $\mathrm{x}$ rebanho nos modelos foi um dos fatores responsáveis pela correlação negativa entre o efeito genético aditivo direto e o materno. Essa dedução foi verificada pela obtenção de correlações próximas de zero e positivas quando se considerou, no modelo, o efeito da interação touro x rebanho.

Tabela 2 - Estimativas dos componentes de (co)variância e parâmetros genéticos para ganho de peso diário do nascimento à desmama (GPND), da desmama aos 365 dias (GPDA) e dos 365 aos 550 dias (GPAS)

Table 2 - Estimates of (co)variance components and genetic parameters for average daily gains from birth to weaning (WBWG), from weaning to 365 days (365WWG) and from 365 to 550 days of age (550YWG)

\begin{tabular}{lcccccc}
\hline $\begin{array}{l}\text { Pesos } \\
\text { Weights }\end{array}$ & $\sigma^{2}{ }_{\mathrm{A}}^{1}$ & $\sigma^{2}{ }_{\mathrm{M}}{ }^{2}$ & $\mathrm{Cov}_{\mathrm{AM}}{ }^{3}$ & $\mathrm{~h}^{2}{ }_{\mathrm{A}}{ }^{4}$ & $\mathrm{~h}^{2}{ }_{\mathrm{M}}{ }^{5}$ & $\mathrm{r}_{\mathrm{AM}}{ }^{6}$ \\
\hline GPND & 0,0010 & 0,0024 & $-0,0012$ & $0,12 \pm 0,04$ & $0,29 \pm 0,09$ & $-0,77$ \\
$\begin{array}{l}\text { WBWG } \\
\text { GPDA }\end{array}$ & 0,0062 & 0,000 & $-0,0001$ & $0,42 \pm 0,12$ & 0,000 & $-0,24$ \\
$\begin{array}{l}\text { 365WWG } \\
\text { GPAS }\end{array}$ & 0,0027 & 0,000 & $-0,0001$ & $0,21 \pm 0,12$ & 0,000 & $-0,44$ \\
550YWG & & 0,000 &
\end{tabular}

1 = variância genética aditiva direta; 2 = variância genética aditiva materna; 3 = co-variância entre efeito genético direto e materno; 4 $=$ herdabilidade para efeito genético aditivo direto; 5 = herdabilidade para efeito genético aditivo materno; $6=$ correlação genética entre efeito genético aditivo direto e materno.

1 = direct additive genetic variance; 2 = maternal additive genetic variance; 3 = covariance between maternal and direct genetc effects; $4=$ heritability for direct additive genetic effects; 5 = heritability for maternal additive genetic effects; $6=$ genetic correlation between maternal and direct additive genetic effects. 
Tabela 3 - Estimativas de herdabilidade para efeito genético aditivo direto (diagonal) e correlações genéticas entre as características estudadas (fora da diagonal)

Table 3 - Heritability estimates for direct additive genetic effects (diagonal) and genetic correlations (out of diagonal)

\begin{tabular}{|c|c|c|c|}
\hline $\begin{array}{l}\text { Características } \\
\text { Traits }\end{array}$ & $\begin{array}{l}\text { GPND } \\
W B W G\end{array}$ & $\begin{array}{c}\text { GPDA } \\
365 W W G\end{array}$ & $\begin{array}{c}\text { GPAS } \\
550 Y W G\end{array}$ \\
\hline $\begin{array}{l}\text { GPND } \\
W B W G\end{array}$ & 0,12 & 0,44 & 0,22 \\
\hline $\begin{array}{l}\text { GPDA } \\
365 W W G\end{array}$ & & 0,42 & 0,30 \\
\hline $\begin{array}{l}\text { GPAS } \\
550 Y W G\end{array}$ & & & 0,21 \\
\hline
\end{tabular}

\section{Conclusões}

As características estudadas estão intrinsecamente associadas aos efeitos ambientais externos (mês e ano de nascimento) e internos (sexo do animal), como também às condições impostas pelos criadores (fazenda). Essas informações subsidiam o produtor, permitindo introduzir mudanças e promover correções no sistema de produção.

A seleção massal baseada no ganho em peso no período de um ano até um ano e meio de idade pode promover considerável progresso genético.

$\mathrm{O}$ efeito materno deve ser incluído nos modelos de avaliação genética dos animais, na característica ganho médio diário do nascimento à desmama, e fazer parte do objetivo de seleção.

\section{Literatura Citada}

FNP-ANUAlPEC. Anuário da Pecuária Brasileira. São Paulo. 1999. 447p.

BIFFANI, S. Influência de fatores ambientais sobre o crescimento de bovinos da raça nelore e estimativa de parâmetros genéticos pelos métodos Henderson 3 e REML Fortaleza: Universidade Federal do Ceará, 1997. 121p. Dissertação (Mestrado em Zootecnia Universidade Federal do Ceará, 1997.

BOLDMAN, K.G.; KRIESE, L.A.; Van VLECK, D.L. et al. A manual for use of MTDFREML. A set of programs to obtain estimates of variances and covariances [DRAFT] Lincoln: USDA/ARS, 1995. 120p.
ELER, J.P.; FERRAZ, J.B.S.; SILVA, P.R. Parâmetros genéticos para peso, avaliação visual e circunferência escrotal na raça Nelore, estimado por modelo animal.Arquivo Brasileiro de Medicina Veterinária e Zootecnia, v.48, p.203-213, 1996.

ELER, J.P.; FERRAZ, J.B.S.; GOLDEN, B.L. et al. Influência da interação touro $\mathrm{x}$ rebanho na estimação da correlação entre efeitos genéticos direto e materno em bovinos da raça Nelore. Revista Brasileira de Zootecnia, v.29, n.6, p.16421648, 2000.

LÔBO, R.B. Programa de melhoramento genético da raça Nelore . 2.ed. Ribeirão Preto: Universidade de São Paulo, 1992.

LÔBO, R.B., BORJAS, A. De los R.; BEZZRRA, L.A. et al. Parâmetros fenotípicos e genéticos de pesos e perímetro escrotal às idades-padrão em animais da raçanelore. In: REUNIÃO ANUAL DA SOCIEDADE BRASILEIRA DE ZOOTECNIA, 32., 1995, Brasília. Anais... Brasília: Sociedade Brasileira de Zootecnia, 1995. p.625-627.

MARTINS FILHO, R.; LÔBO, R.B.; LIMA, F.A.M. et al. Parâmetros genéticos e fenotípicos de pesos e ganhos em pesos de bovinos zebus no Estado do Ceará. In: REUNIÃO ANUAL DA SOCIEDADE BRASILEIRA DE ZOOTECNIA, 34., 1997, Juiz de Fora.Anais...Juiz de Fora: Sociedade Brasileira de Zootecnia, 1997. p.248-250.

MARTINS, G.A.; MARTINS FILHO, R.; LIMA, F.A.M.; LÔBO, R.N.B. Influência de fatores genéticos e de meio sobre o crescimento de bovinos nelore no Estado do Maranhão. In: REUNIÃO ANUAL DA SOCIEDADE BRASILEIRA DE ZOOTECNIA, 35., 1998, Botucatu. Anais... Botucatu: Sociedade Brasileira de Zootecnia, 1998. p.431- 433.

MAGNABOSCO, C.U.; FAMULA, T.R.; LÔBO, R.B. et al. Estimativas de parâmetros genéticos e de ambiente de características de crescimento em bovinos da raça Nelore. In: REUNIÃO ANUAL DA SOCIEDADE BRASILEIRA DE ZOOTECNIA, 33., 1996, Fortaleza. Anais... Fortaleza: Sociedade Brasileira de Zootecnia, 1996. p.142-144.

MUNIZ, C.A.S.D.; QUEIROZ, S.A. Avaliação do peso à desmama e do ganho médio de peso de bezerros cruzados, no Estado do Mato Grosso do Sul. Revista Brasileira de Zootecnia, v.27, n.3, p.504-512, 1998.

SAS STATISTICAL ANALYSIS SYSTEM. User's guide: Statistics. Version 6.11, NC; SAS Institute, 1996.

SNYMAN, M.A.; ERASMUS, G.J.; Van WIK, J.B. Non genetic factors influencing growth and fleece traits in Afrino sheep. South African Journal of Animal Science, v.25, p.55-62, 1995.

SOUSA, W.H.; PEREIRA, C.S.; BERGMAN, J.A.G. et al. Estimativas de $(\mathrm{Co})$ variâncias e herdabilidades direta e materna de pesos corporais em ovinos da raça Santa Inês. Revista Brasileira de Zootecnia, v.28, n.6, p.1252$1262,1999$.
Recebido em: $12 / 11 / 01$

Aceito em: 08/10/02 